\title{
Going against the flow: effects of non-Gaussian dispersal kernels and reproduction over multiple generations
}

\author{
J. M. Pringle ${ }^{1, *}$, F. Lutscher ${ }^{2}$, E. Glick ${ }^{3}$ \\ ${ }^{1}$ Ocean Process Analysis Laboratory, University of New Hampshire, Durham, New Hampshire 03824, USA \\ ${ }^{2}$ Department of Mathematics and Statistics, University of Ontario, 585 King Edward Ave, Ottawa, Ontario K1N 6N5, Canada \\ ${ }^{3}$ Bryn Mawr College, Bryn Mawr, Pennsylvania 19010, USA
}

\begin{abstract}
Our previous studies (Byers \& Pringle 2006; Mar Ecol Prog Ser 313:27-41, Pringle \& Wares 2007; Mar Ecol Prog Ser 335:69-84) discuss when populations and gradients in allele frequency can persist in the presence of a mean downstream dispersal of propagules from the parents' location. These studies assume that reproduction is uniform over the lifetime of the adult and that the larval dispersal kernel is 'nearly' Gaussian in shape. These results are extended in the present study to include variable reproduction over the lifetime of an adult and non-Gaussian dispersal kernels. It is found that persistence is governed by the lifetime reproductive output of the adults. The impact of non-Gaussian dispersal kernels is quantified in terms of the excess kurtosis of the dispersal kernel.
\end{abstract}

KEY WORDS: Advection · Retention · Planktonic larvae · Coastal · Physical-biological coupling · Dispersal

Resale or republication not permitted without written consent of the publisher

\section{INTRODUCTION}

Byers \& Pringle (2006) and Pringle \& Wares (2007) (hereafter BWP) discuss when populations and gradients in allele frequency can be retained in the presence of a mean downstream dispersal of propagules from the parents' location, as occurs, for example, in benthic species in the coastal ocean. However, in their analysis they make a number of assumptions which they do not fully justify. In particular, they assert that it is the lifetime fecundity of the species in the absence of density dependence that governs the persistence of the species and alleles; however, they only test this result for the case in which reproductive output is constant with time over the lifetime of the organism. This is clearly not always a good assumption; many invertebrate marine species produce more larvae as the adults grow older and larger (Llodra 2002). These authors also assume a Gaussian dispersal kernel and assure the reader that any kernel that is 'close to a Gaussian' will lead to similar results to those obtained with a
Gaussian kernel. Unfortunately, they do not define what 'close' means, thus leaving the reader uncertain as to how to apply the results to real world dispersal kernels. Thus, the reader is unsure what to do when; to use an example from Byers \& Pringle (2006), a species reproduces in multiple seasons in which the mean and variability of the currents are different, so that even if the dispersal kernel for each reproductive event is Gaussian, the net larval dispersal kernel is a composite of the distribution for each spawning event and will not be Gaussian.

In the following section, we will provide 2 results that address these issues. First, we will show that the criteria for retention of a species is, for a Gaussian dispersal kernel,

$$
\frac{L_{\text {adv }}^{2}}{2 L_{\text {diff }}^{2}}<\ln (N)
$$

where $N$ is the total number of larvae which would recruit and reach reproductive competency in the absence of density-dependent effects per adult per 
lifetime. $L_{\mathrm{adv}}$ and $L_{\text {diff }}$ are the mean and standard deviation of the larval dispersal distance, as discussed in BWP. This is the same as Eq. (6) of Byers \& Pringle (2006) when the reproductive output of the adults is constant with age, but is also correct when the reproductive output of the adult varies with age. Second, we show that if the kernel is non-Gaussian, the criteria for retention becomes approximately

$$
\frac{L_{\text {adv }}^{2}}{2 L_{\text {diff }}^{2}}<\ln (N)+\frac{\gamma_{2}}{6} \ln (N)^{2}+\frac{\gamma_{2}^{2}}{144} \ln (N)^{3}
$$

where $\gamma_{2}$ is the 'excess kurtosis', and is equal to $\mu_{4} / L_{\text {diff }}^{4}-3$, where $\mu_{4}$ is the fourth central moment of the kernel. The kurtosis is a measure of how many of the larvae of a given kernel are in the edges of the dispersal kernel relative to the center of the kernel for a given standard deviation of larval dispersal distance, $L_{\text {diff. }}$. This can be seen in the 2 panels of Fig. 1, where the kernels with larger and more positive kurtosis have more larvae in their tails and, in order to keep the standard deviation constant, more larvae concentrated at the center of the distribution. Excess kurtosis is defined with respect to a Gaussian distribution, so that a positive excess kurtosis indicates more larvae in the tails of the distribution than a Gaussian kernel would have, and a negative value indicates fewer. Thus, a Gaussian kernel has an excess kurtosis $\gamma_{2}$ of zero, and the above criterion reduces to that of BWP for that kernel. The excess kurtosis for other kernels can be found in Lutscher (2007). This criterion for retention can be used to judge how important the deviations from a Gaussian kernel are, and, if the effect is large, numerical methods can then be used to calculate the exact retention criterion (the code to do so is available in the online supplement to this article; Appendix 1, www.int-res.com/articles/ suppl/m377p013_app/). This result can be transferred to Pringle \& Wares (2007) by substituting the right-hand side of Eq. (2) for the right-hand side of Eqs. (3), (4) and (10) in that paper. It is also shown below that when there is no mean downstream dispersal of larvae, the excess kurtosis does not affect the persistence of a species, consistent with Lockwood et al. (2002).

The impact of non-Gaussian kernels, such as those shown in Fig. 1, can be very important when there is a mean downstream transport of larvae. In Fig. 2, the critical value of $N$ needed to allow retention is shown as a function of the mean larval dispersal distance $L_{\text {adv }}$ for these 3 non-Gaussian dispersal kernels, each representative of a certain kind of deviation from an idealized Gaussian kernel. For each kernel, the standard deviation of the dispersal distance of successfully recruiting larvae $L_{\text {diff }}$ is $30 \mathrm{~km}$ while the mean dispersal distance varies from 1 to $50 \mathrm{~km}$. The first kernel is the composite of 2 Gaussian kernels, such as might occur if the species spawns in 2 different seasons with

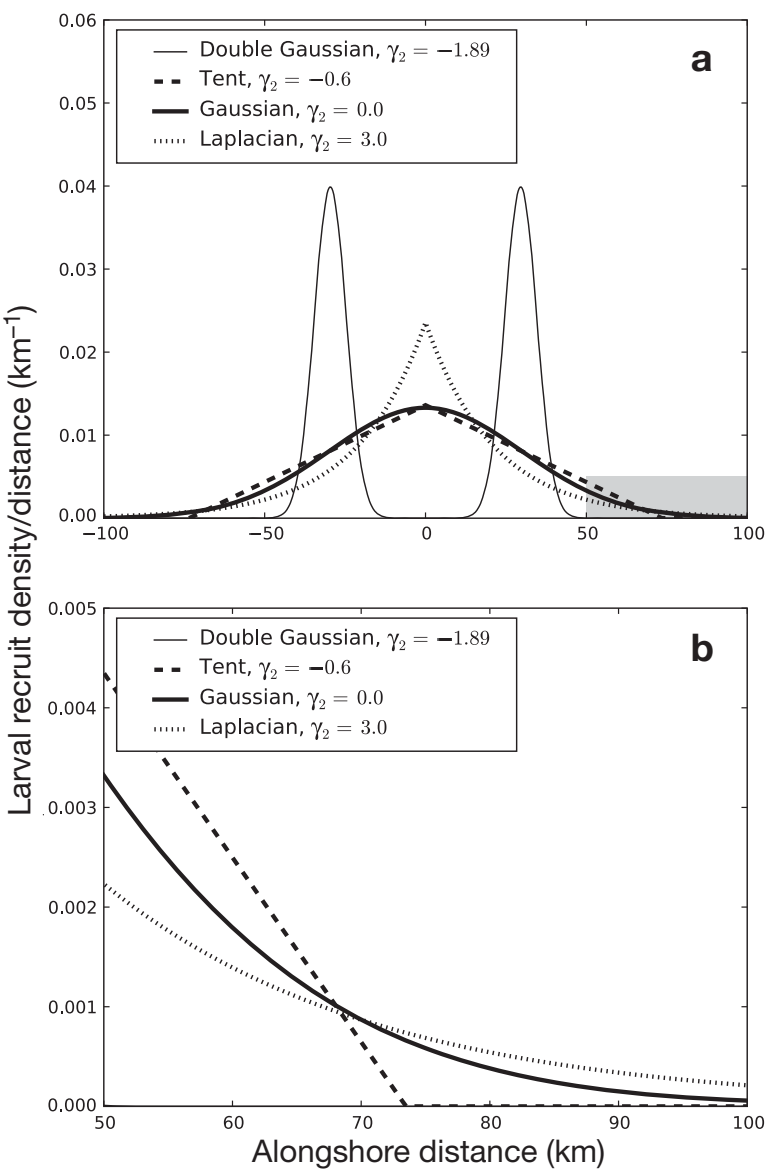

Fig. 1. (a) The 4 larval dispersal kernels examined in Fig. 2. For each, the standard deviation of the distance the larvae disperse, $L_{\text {diff, }}$ is $30 \mathrm{~km}$, while the mean distance $L_{\text {adv }}$ is 0 in these plots (though not in Fig. 2). (b) The right-hand tails of the same kernels, expanded from the shaded portion of (a). The double Gaussian kernel's tail is too close to zero to be seen on this scale. $\gamma_{2}$ : excess kutosis

different mean currents. This has a negative $\gamma_{2}$ of -1.89 , indicating that the tails of the dispersal kernel are relatively small for a given value of $L_{\text {diff. }}$ Thus, fewer larvae settle far from the center of the larval recruitment distribution, increasing the $N$ needed to allow retention above the prediction of for a given $L_{\text {adv }}$ and $L_{\text {diff. }}$. The second kernel is tent shaped and there is no dispersal outside of a finite distance. Its excess kurtosis is $\gamma_{2}=-0.6$. This models the truncation of the tails of the dispersal kernel which can occur because there is a practical upper limit on the speed of the currents in the ocean, and thus a limit to the dispersal distance of a larvae. This tends to increase the $N$ needed to allow retention relative to the Gaussian prediction by eliminating the rare long-distance dispersal of larvae. The third set of results are for a Gaussian kernel, for which $\gamma_{2}=0$ and the results of BWP and Eq. (2) agree. The last kernel is a Laplacian kernel, with $\gamma_{2}=3.0$. With this kernel, the density of larval recruitment is increased 

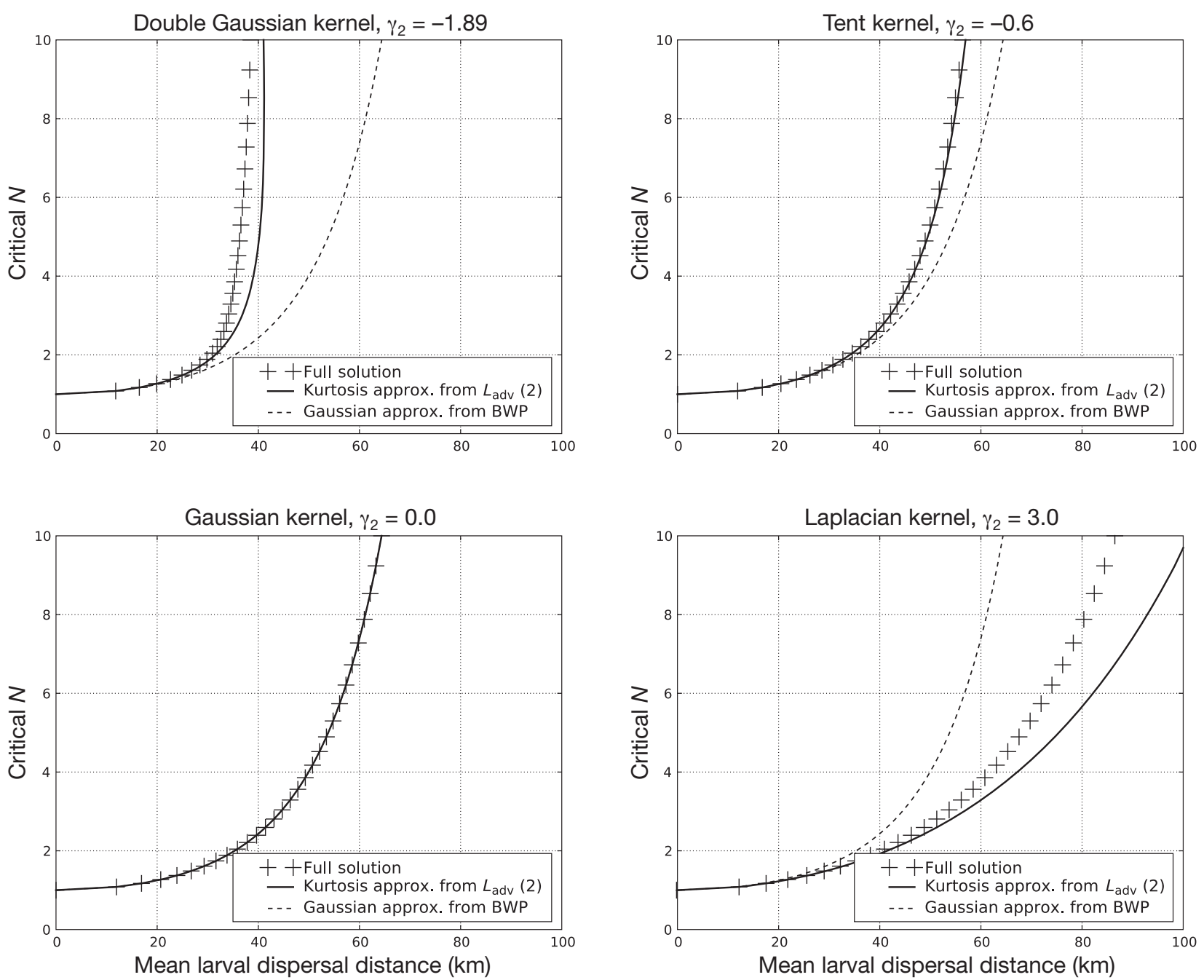

Fig. 2. The critical value of $N$ needed to allow the retention of a population as a function of the mean distance the larvae disperse, $L_{\mathrm{adv}}$, for the various dispersal kernels shown in Fig. 1. $N$ : total number of larvae which would recruit and reach reproductive competency in the absence of density-dependent effects per adult per lifetime. Shown is the true value, computed numerically as described in the text, the estimate which includes the effect of the excess kurtosis $\left(\gamma_{2}\right)$ of the dispersal kernel from Eq. (2), and the estimate assuming a Gaussian kernel from Byers \& Pringle (2006) and Pringle \& Wares (2007) (BWP). Plots are ordered from least excess kurtosis, the Double Gaussian kernel with $\gamma_{2}=-1.89$, to the greatest excess kurtosis, the Laplacian kernel with $\gamma_{2}=3.0$

both near the center of the larval distribution and far from the center, leading to a sharply peaked distribution with long tails of dispersal distance. These tails tend to increase the ability of larvae to be retained for a given fecundity and recruitment rate $N$ relative to an equivalent Gaussian kernel.

For each non-Gaussian kernel, the approximation in Eq. (2) is more accurate than the criteria in BWP calculated under the assumption of a Gaussian kernel. It successfully captures the increase in $N$ needed for retention when $\gamma_{2}<0$, and the decrease when $\gamma_{2}>0$. Further discussion of the dynamics behind these results is given in the context of invasion speeds in Lutscher (2007). When the magnitude of the excess kurtosis of the dispersal kernel or $L_{\text {adv }}$ becomes large, the approximate formula (Eq. 2) becomes increasingly inaccurate. In practice, it would be prudent to use Eq. (2) to estimate the effect of the excess kurtosis on the critical value of $N$ needed to allow retention and, if this effect is large, then to calculate the exact critical value of $N$ numerically using the code in Appendix 1.

The following sections lay out the derivations of these results, but present no further results.

\section{RETENTION AND REPRODUCTION}

Neubert \& Caswell (2000) derive a method for determining the invasion speed of a population in a stagestructured population given any dispersal kernel with 
exponentially bounded tails. They argue that it is not necessary to include density-dependent effects in this calculation, as long as there are no allee or longdistance density-dependence effects. We assume discrete generations of an organism that only disperses when spawned, and is afterwards sessile, as in BWP. Following Neubert \& Caswell (2000), and assuming that the dispersal kernel $K$ is independent of the parents location and includes the mean transport of the larvae, the population at location $x$ and time $t+1$ in generations, $\mathbf{p}(x, t+1)$, is given by the convolution:

$$
\mathbf{p}(x, t+1)=\int_{-\infty}^{\infty} \mathrm{d} y[\mathbf{A}(x-y) \mathrm{p}(y, t)]
$$

Here, $\mathbf{p}(x, t)$ is a vector whose elements are the population age structure, $y$ is a dummy variable of integration, and $\mathbf{A}$ is the matrix that describes how the larval settlement is distributed in space and how each generation ages (the combined $\mathbf{K} \bigcirc \mathbf{B}$ of Neubert \& Caswell (2000)):

$$
\mathbf{A}=\left[\begin{array}{cccc}
N_{1} K & N_{2} K & \ldots & N_{n} K \\
\delta r_{1} & 0 & \ldots & 0 \\
0 & \delta r_{2} & \ldots & 0 \\
\vdots & & \ddots & \vdots \\
0 & 0 & \ldots & 0
\end{array}\right]
$$

Subscripts indicate the age in generations, $r_{i}$ is the likelihood that an individual of age $i$ will live to $i+1, N_{i}$ is the fecundity of an adult of age $i, n$ is total number of generations an organism can live, $\delta$ is the Dirac delta function, indicating that adults are sessile, and both $K$ and $\delta$ are functions of $(x-y)$. (In BWP, $N_{i}$ is uniform for each generation and is called $N_{\text {fec.) }}$ ) Neubert \& Caswell (2000) show that the invasion speed can be found from the matrix formed by computing the moment generating function of each element of $\mathbf{A}$, which is calculated by multiplying each element by $e^{s x}$, and integrating over $x$ from $-\infty$ to $\infty$. This results in

$$
\mathbf{B}=\left[\begin{array}{cccc}
N_{1} M(s) & N_{2} M(s) & \ldots & N_{n} M(s) \\
r_{1} & 0 & \ldots & 0 \\
0 & r_{2} & \ldots & 0 \\
\vdots & & \ddots & \vdots \\
0 & 0 & \ldots & 0
\end{array}\right]
$$

where $M(s)$ is the moment generating function of the dispersal kernel $K$.

If the mean dispersal is towards smaller $x_{1}$ the upstream dispersal speed $C$ is given by the minimum

$$
C=\min _{s>0}\left\{\frac{1}{s} \ln [\rho(s)]\right\}
$$

as a function of $s$, where $\rho(s)$ is the largest eigenvalue of B (Neubert \& Caswell 2000). Now, at the critical value of population growth that just allows a population to be retained, the upstream invasion speed must be zero. If the invasion speed is negative, the population is being washed downstream, and if it is greater than zero, the population has more than enough growth to persist (BWP). Thus, at the critical growth rate for retention, $\rho(s)$ must be 1 . The largest eigenvalue of the matrix $\mathbf{B}$ is given by largest root of the characteristic polynomial (Strang 1988)

$$
\begin{aligned}
& \rho^{n}-N_{1} M(s) \rho^{n-1}-r_{1} N_{2} M(s) \rho^{n-2}-r_{1} r_{2} N_{3} M(s) \rho^{n-3} \\
& \ldots-\left(r_{1} r_{2} \ldots r_{n-1}\right) N_{n} M(s)=0
\end{aligned}
$$

Setting $\rho=1$, and assuming that $K$ and thus $M(s)$ does not vary with the age of the parents, the critical condition of $C=0$ becomes

$$
\sum_{j=1}^{n}\left\{\left(\prod_{l=1}^{j-1} r_{l}\right) N_{j}\right\} M(s)=1
$$

The sum of the products above is just the likelihood that an adult reaches age $j, \prod_{l=1}^{j-1} r_{l}$, multiplied by the reproductive success for that age, $N_{j}$, i.e. it is just the expected total reproduction of an individual $N$, neglecting density-dependence effects. Thus, the minimum total lifetime reproduction needed for retention is given when Eq. (8) is satisfied for the smallest value of $M(s)$, i.e.

$$
N \min _{s>0} M(s)=1
$$

This condition depends only on the dispersal kernel (which sets $M(s)$ ) and $N$, regardless of how the fecundity of the organism varies with age. Thus, retention is governed by the expected total number of larvae which would settle and reach reproductive competency in the absence of density-dependent effects over the lifetime of an adult, $N$, and the dispersal kernel. For the Gaussian kernel with mean dispersal distance $L_{\mathrm{adv}}$, the moment generating function is given by:

$$
M(s)=\exp \left(L_{\mathrm{adv}} s+L_{\mathrm{diff}}^{2} s^{2} / 2\right)
$$

Solving Eq. (9) with Eq. (10), we obtain Eq. (1) for species with Gaussian dispersal kernels. We can recover the results of Byers \& Pringle (2006) by noting that their $N_{\text {fec }}$ is $N_{i}$ above and is the same for each generation, their $N_{\text {gen }}$ is $n$, and they assume $r_{i}=1$. In these limits, $N_{\text {fec }} N_{\text {gen }}=N$, and so their criteria for retention in iteroparous species reduces to Eq. (1).

\section{NON-GAUSSIAN KERNELS}

For any arbitrary kernel, the critical value of $N$ or $L_{\text {adv }}$ needed to allow retention can be found from Eq. (9). However, while straightforward to do so computationally, it is difficult to make analytical headway in this manner. Instead, we take advantage of 2 results. First, Pachepsky et al. (2005) note that the criterion for retention of a species with a mean larval dispersal dis- 
tance per generation $\left(L_{\mathrm{adv}} \neq 0\right)$ can be found by computing the upstream invasion distance per generation that would result from its dispersal kernel in the absence of a mean downstream dispersal $\left(L_{\mathrm{adv}}=0\right)$. If this invasion speed exceeds the mean dispersal distance per generation, the species can be retained. Secondly, Lutscher (2007) computes an approximate invasion speed for an arbitrary dispersal kernel which has no mean downstream dispersal. When Lutscher (2007)'s invasion speed in distance per generation exceeds the $L_{\mathrm{adv}}$, the population will persist.

Following Weinberger (1982), we note that the invasion speed for an semelparous organism with $L_{\mathrm{adv}}=0$ is given by:

$$
C=\min _{s>0} \frac{1}{S} \ln [N M(s)]
$$

where $M(s)$ is the moment generation function of the kernel discussed above. (This is just Eq. (6) written for the semelparous case. As shown in the last section, the semelparous results will also be applicable to the iteroparous case.) $M(s)$ can be expanded in the raw moments of the dispersal kernel $M(s)=1+\sum_{i=1 \ldots \infty} \frac{s^{i}}{i !} \mu_{i}^{\prime}$. Since the mean downstream transport of the larvae in this calculation is zero, the first raw moment is zero, and we shall assume that the dispersal kernel is not skewed, so that the third raw moment is zero. Keeping the expansion to $O\left(s^{4}\right)$ in $s$ of $M(s)$, making a Taylor series expansion of $\ln (N M(s))$ in Eq. (11) to the same order, and finding the minimum of this expansion, leads to an estimate of the upstream invasion speed in units of distance per generation $C^{*}$ of:

$$
C^{*}=\sqrt{2 L_{\text {diff }}^{2} \ln N}\left(1+\frac{\gamma_{2}}{12} \ln N\right)
$$

where $\gamma_{2}$ is the excess kurtosis as defined in the introduction. When $C^{*}$ is greater than $L_{\mathrm{adv}}$, the species can persist. Solving for this criterion leads to the expression given in Eq. (2).

Eq. (2) provides an estimate of how the excess kurtosis of a dispersal kernel can alter the estimates of the population growth rate needed to allow a population to persist. However, if one is using dispersal kernels derived either from observation or numerical model, one is likely to find the kernel has non-negligible skew

Editorial responsibility: Steven Morgan,

Bodega Bay, California, USA (the third central moment of the dispersal kernel), and may have higher moments that further modify the persistence criterion away from Eq. (2). However, it is straightforward to compute the persistence criteria numerically, and in the supplemental online material for this article there is a Python program to compute the persistence criteria for an arbitrary dispersal kernel. This code directly solves Eq. (11) for $c=0$ for a user-defined dispersal kernel.

Excess kurtosis only affects population persistence when there is a mean downstream dispersal of larvae (e.g. $L_{\mathrm{adv}}=0$ ), as has been pointed out by, among others, Lockwood et al. (2002). These results are consistent with Eq. (2), for when $L_{\mathrm{adv}}=0$, the criterion for persistence becomes $\ln (N)>0$ or, equivalently, $N>1$. When $\ln (N)$ approaches 0 , the terms involving the excess kurtosis in Eq. (2) approach zero, and thus no longer affect the criterion.

Acknowledgements. John Wares and Jeb Byers provided some of the biological underpinnings and examples for this paper. This work was funded by NSF grant OCE-0453792 to J. M. P. and the NASA-UNH Research and Discover Program to E. G.

\section{LITERATURE CITED}

Byers JE, Pringle JM (2006) Going against the flow: retention, range limits and invasions in advective environments. Mar Ecol Prog Ser 313:27-41

Llodra ER (2002) Fecundity and life-history strategies in marine invertebrates. Adv Mar Biol 43:87-170

Lockwood DR, Hastings A, Botsford LW (2002) The effects of dispersal patterns on marine reserves: Does the tail wag the dog? Theor Popul Biol 61:297-309

Lutscher F (2007) A short note on short dispersal events. Bull Math Biol 69:1615-1630

Neubert MG, Caswell H (2000) Demography and dispersal: calculation and sensitivity analysis of invasion speed for structured populations. Ecology 81:1613-1628

Pachepsky E, Lutscher F, Nisbet RM, Lewis MA (2005) Persistence, spread and the drift paradox. Theor Popul Biol 67: $61-73$

Pringle JM, Wares JP (2007) Going against the flow: maintenance of alongshore variation in allele frequency in a coastal ocean. Mar Ecol Prog Ser 335:69-84

Strang G (1988) Linear algebra and its applications, 3rd edn. Brooks/Cole, New York

- Weinberger HF (1982) Long-time behavior of a class of biological models. SIAM J Math Anal 13:353-396

Submitted: May 22, 2008; Accepted: November 17, 2008

Proofs received from author(s): February 1, 2009 